\title{
Modeling and Control of wind magnetic Suspension Yaw Motor
}

\author{
Dengpeng Guo ${ }^{1}$, Xiaoguang $\mathrm{Chu}^{1,3}{ }^{1,} \mathrm{a},{ }^{*}$ Bin $\mathrm{Cai}^{1}$, Ying Kong ${ }^{2,4}$, Naizhe Wang ${ }^{1}$ \\ and Xuetao $\mathrm{Yi}^{1}$ \\ ${ }^{1}$ Qufu Normal University engineering college Shandong China \\ 2 Jining Medical University Shandong China \\ ${ }^{3}$ National Natural Science Foundation of 61473170 China \\ ${ }^{4}$ Jining Science and Technology Development Plan (soft) 20146221 China \\ axg_chu@163.com
}

Keywords: Modeling, Control, wind magnetic.

\begin{abstract}
The magnetic levitation technique is introduced into the wind yaw system to reduce the yaw power. Permanent magnet in the nacelle and stator winding in tower are designed to suspend the nacelle. And the wind yaw structure including the disc motor rotor and variable speed gear is proposed to drive suspension nacelle for wind direction. Based on the nacelle suspension mechanism, the disc rotor excitation current model is constructed, and then the nacelle yaw load torque model is given with the consideration of the wind and the nacelle suspension state, and then the whole dynamic model of the yaw disc motor is finished. In view of nonlinear and the high disturbance, the sliding mode speed controller is designed with the load torque observer to acquire the current reference, and then two PI current controllers are designed to realize wind yawing for wind direction. Based on simulation platform, the wind suspension yaw structure and the control strategy are verified with the stable nacelle suspension air gap and the yaw speed under the variation of load torque.
\end{abstract}

\section{Introduction}

Wind power has been the priority direction of power development strategy in the developed countries. Yaw system is the important component for the maximum wind energy. The failure rates of traditional wind yaw system are high to $20 \%$ because of the complex structure ${ }^{[1]}$. When the yaw system comes to failure, the yaw system maintenance must be relied on the crane, ships, helicopters and other large equipment. It is urgent to study the novel yaw system with the easy maintenance and high reliability. To this end, the project proposed a new wind turbine yaw system that using maglev drive technology (as shown in figure 1). Through the electromagnetic suspension, the nacelle is in suspension state, the friction loss is reduced. And the yaw system structure will be simplified, the failure rate is reduced.

\section{Operation Mechanism of Maglev Yaw System}

As shown in Figure 1, the novel wind magnetic levitation yaw system is proposed to reduce the nacelle yaw power and improve the accuracy of the yawing for wind direction. The key device is a special structure of the disc synchronous motor, whose stator is located in the tower-integrated structure. And 16 DC windings are arranged, to provide the excitation current of disc motor and suspend the nacelle for the decrease of the yaw load torque. The disc motor rotor is installed inside the tower and mechanically coupled with the gears to drive the nacelle yaw for direction. 


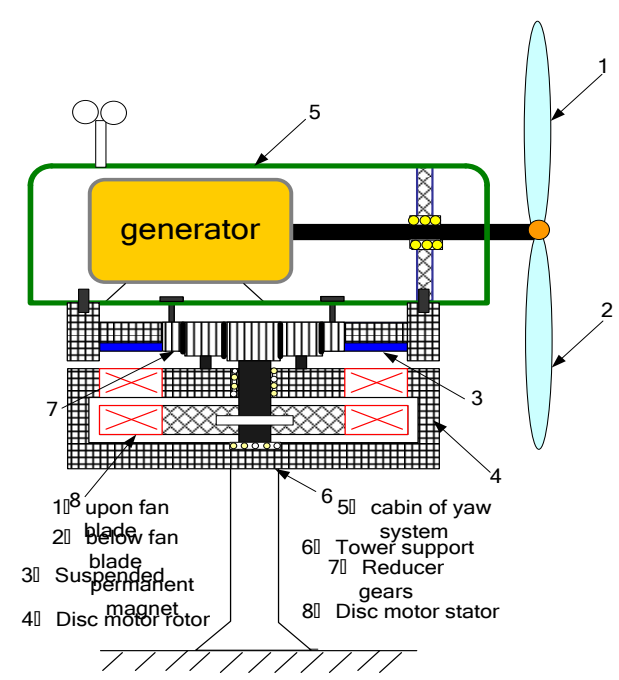

Figure 1. Magnetic levitation yaw system

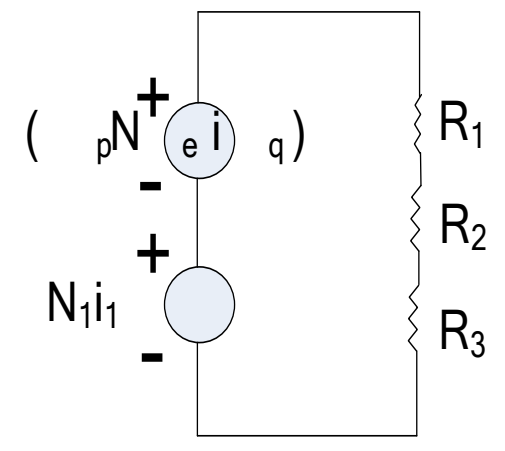

Figure 2. Suspension equivalent

When wind direction changes, the magnetic levitation yaw system is activated, stator windings are powered to produce the suspension force with the permanent magnets to suspend the nacelle. The difference of the excitation current affects the state of nacelle suspension, which decides the yawing friction loss. When the nacelle is completely suspended, the yawing friction loss will decrease to zero, and the yaw load torque ${ }^{[2]}$ will also decrease much more, but the main part is the nacelle rotation torque which is seriously affected by the wind, yaw load torque is still much and can be expressed as:

$$
\begin{gathered}
T_{L}=T_{c f}+T_{s}+T_{f} \\
T_{c f}=\left(m g+\frac{1}{2} C_{n} \rho(v \sin \beta)^{2} A_{n}\right) R_{n} \\
T_{s}=\frac{1}{2} C_{s} \rho(v \sin \theta)^{2} A_{s} L \\
T_{f}=\mu_{0} F R=\mu_{0}\left(m g+f_{d}-F_{M}\right) R_{n}
\end{gathered}
$$

Where $T_{L}$ is the load torque; $T_{\text {cf }}$ for the rotational torque; $T_{s}$ for the wind resistance torque; $T_{\mathrm{f}}$ for the friction torque; $m$ for the nacelle weight; $g$ for the acceleration of gravity; $C_{n}$ for the wind energy utilization coefficient; $\rho$ for the air density; $v$ for the wind speed; $\beta$ for the wind speed pitch angle; $A_{n}$ for the nacelle side of the effective area; $R_{n}$ for the nacelle radius; $C_{s}$ for the lateral wind power; $\theta$ for the yaw angle; $A_{s}$ for the fan blade sweep area; $L$ for the nacelle yaw arm; $\mu_{0}$ for the friction coefficient; $F$ for the upside pressure together; $f_{d}$ for the Under pressure; $F_{M}$ for the electromagnetic levitation force.

In order to ensure the safety and stability of the yaw operation ${ }^{[3]}$, the yaw speed must be strictly controlled in the safety range. The maximum yaw speed must meet the following relationship:

$$
\omega_{\text {yaw, } \max }=\left[3-2\left(\frac{A_{s}-2}{200-2}\right)\right] \mathrm{rad} / \mathrm{s}\left(A_{s}>2 \mathrm{~m}^{2}\right)
$$

Wind blade sweep area of $50 \mathrm{~kW}$ is about $81 \mathrm{\pi m}^{2}$, so the yaw speed is set $1 \mathrm{r} / \mathrm{min}$ for yaw work safety. A gear (Variable ratio 100:1) is set to reduce the coupling relationship of the nacelle suspension and yawing torque, to achieve yaw motor rotor speed 100r/min deceleration processing.

Remarks: Yaw disc motor excitation current determines the load torque, when the excitation current is small, the suspension force cannot achieve the nacelle suspension. In this paper, the nacelle is suspended with a stable air gap of $0.01 \mathrm{~m}$. However, the variation of yaw angle and wind speed fluctuation easily lead to the fluctuation of air gap, which seriously affects the stable operation of yaw process. Therefore, the magnetic levitation yaw type motor control during the load changes is meaningful.

\section{Dynamic Model of Disk Motor}

The magnetic levitation yaw motor is an external excitation synchronous motor with 8 pairs of poles. In the process of modeling of the disc yaw, the suspension model is first constructed.

Levitation force ${ }^{[4]}$ is first analyzed, and suspension equivalent diagram is shown in figure 2. 
The reluctance satisfies the following relationship:

$$
R_{1}=\frac{x_{11}}{\mu_{1} w_{1} d_{1}} \quad R_{2}=\frac{h_{2}}{\mu_{2} w_{2} d_{2}} \quad R_{3}=\frac{h_{3}}{\mu_{3} w_{3} d_{3}}
$$

According to the energy conservation law the available levitation force is obtained as follows:

$$
F_{M}=-\frac{1}{4} \frac{\mu_{1} s_{1}\left(N_{1}^{2} i_{1}^{2}+N^{2} i^{2}\right)}{\left[x_{11}+\mu_{1} s_{1}\left(\frac{h_{2}}{\mu_{2} s_{2}}+\frac{h_{3}}{\mu_{3} s_{3}}\right)\right]^{2}}
$$

Where: $x_{11}$ for the variable air gap height, $\mu_{1}$ for the air permeability, $s_{1}=w_{1} d_{1}$ for the suspension equivalent area, $h_{2}$ for the permanent magnet height, $\mu_{2}$ for the permanent magnetic permeability, $s_{2}=w_{2} d_{2}$ for the permanent magnet cross-sectional area, $h_{3}$ for the ferromagnetic material height, $\mu_{3}$ for the ferromagnetic material Permeability, $s_{3}=w_{3} d_{3}$ for the ferromagnetic material cross-sectional area, the negative sign indicates that the suspension winding is attractive.

In view of the levitation force generated by the suspension winding is not only related to the suspended current but also the properties of the ferromagnetic material so it will increase the difficulties of controlling.

According to Newton's second law, the suspension modeling can be expressed as:

$$
\left\{\begin{array}{l}
\ddot{\delta}=\mathrm{g}-\frac{F_{M}(i, \delta)}{m}+\frac{f_{\mathrm{d}}(t)}{m} \\
\frac{d i_{\mathrm{f}}}{d t}=\left[U_{f}-R_{f} i_{f}+\frac{\mu_{1} s_{1} N^{2} i_{e q}}{\delta^{2}(t)} \frac{d \delta}{d t}+f_{t}(t)\right] \frac{h_{3}}{\mu_{3} s_{3} N^{2}}
\end{array}\right.
$$

Which $\delta$ is the suspended air gap; $g$ for the acceleration of gravity; $m$ for the quality of the equipments; $F_{M}(i, \delta)$ for the levitation force of the rotor winding; $U_{f}$ for the DC excitation voltage; $R_{f}$ for the excitation resistance; $i_{f}$ for the excitation current; $f_{t}(t)$ for the system interference.

However, in the steady-state of the suspension, the excitation magnetic field is basically constant in view of the balance between the levitation force and the pressure force. So in the process of modeling of the disc motor, mainly focusing on the stator three-phase voltage dynamic model construction, ignoring the damping windings, and after decoupling transformation the model can be expressed as:

$$
\left[\begin{array}{l}
u_{s d} \\
u_{s q}
\end{array}\right]=\left[\begin{array}{ccc}
R_{s} & -\omega L_{s} & 0 \\
\omega L_{s} & R_{s} & \omega L_{m}
\end{array}\right]\left[\begin{array}{l}
i_{s d} \\
i_{s q} \\
i_{f}
\end{array}\right]+\left[\begin{array}{ccc}
L_{s} & 0 & L_{m} \\
0 & L_{s} & 0
\end{array}\right] p\left[\begin{array}{l}
i_{s d} \\
i_{s q} \\
i_{f}
\end{array}\right]
$$

In view of the part of rotor excitation current changes slower than the speed of the part of stator rotation, so the rotor excitation current can be equivalent to a constant when the stator is in the high-speed rotation process, according to this the yaw disc motor can be further equivalent.

$$
\left\{\begin{array}{l}
\frac{d \omega}{d t}=\frac{n_{p}}{J}\left(T_{e}-T_{L}-\frac{B}{n_{p}} \omega\right) \\
\frac{d i_{s d}}{d t}=-\frac{R_{s}}{L_{s}} i_{s d}+\omega i_{s q}+\frac{1}{L_{s}} u_{s d} \\
\frac{d i_{s q}}{d t}=-\omega i_{s d}-\frac{R_{s}}{L_{s}} i_{s q}-\frac{L_{m}}{L_{s}} \omega i_{\mathrm{f}}+\frac{1}{L_{s}} u_{s q}
\end{array}\right.
$$

Where $\omega 、 i_{s d} 、 i_{s q}$ is the state variable, the $u_{s d} 、 u_{s q}$ is variable of input, $T_{L}$ for the disturbance, $\omega$ for the electrical angular velocity of yaw motor, $J$ for the moment of inertia, $T_{e}$ for the yaw electromagnetic torque.

For detailed modeling of yaw electromagnetic torques:

$$
T_{e}=\frac{3}{2} n_{p}\left\{L_{m} i_{f} i_{s q}+\left(L_{s d}-L_{s q}\right) i_{s d} i_{s q}+\left(L_{m} i_{r d} i_{s q}-L_{m} i_{r q} i_{s d}\right)\right\}
$$

Ignoring the effects of damper windings, the electromagnetic torque of yaw motor can be expressed as: 


$$
T_{e}=1.5 n_{p} L_{m} i_{f} i_{s q}
$$

Through the analysis of the model, it is found that $n_{p}, L_{m}$ and $i_{f}$ can be seen as constant variables so can optimize the current of the stator torque to achieve the target of a constant speed.

\section{Control Strategy of Maglev Yaw Motor}

The control strategy of magnetic levitation yaw system ${ }^{[5]}$ adopts nacelle suspension controller and yaw motor speed controller. The nacelle is suspended by the suspension controller with a constant air gap reference. This paper mainly focuses on the yaw speed control under the stability of air gap. The rotor flux orientation is used to decouple the rotor current with excitation current and the torque current. The Sliding Mode Speed Controller is adopted to online optimize the reference torque current iq, and then the current proportional integral controller is used to track the reference the optimized current to achieve the effective speed control. In view of the external wind fluctuation, the variation of the excitation current and sliding mode chatting, the load observer is proposed to observe these mismatches of the model.

\subsection{Sliding Mode Speed Controller}

Sliding mode control [6] [7] is more suitable for industry applications with the feature of robustness and fine tracking performance, so it can be applied to resolve the high non-linear and interference of the speed yaw control. In order to control the speed to reach the target, define the motor speed as the state variable:

$$
\dot{x}=\frac{1}{J}\left(\frac{3}{2} n_{p} L_{m} i_{f} u-T_{L}\right)
$$

In order to realize the speed control, the integral sliding surface is designed as follows:

$$
s(t)=c e(t)+\dot{e}(t) \quad(c>0)
$$

The speed tracking error and its derivative are defined as:

$$
e(t)=\int \omega(t)-\int \omega_{r e f}(t), \quad \dot{e}(t)=\omega(t)-\omega_{r e f}(t)
$$

Here $\omega_{\text {ref }}(t)$ is the speed reference trajectory.

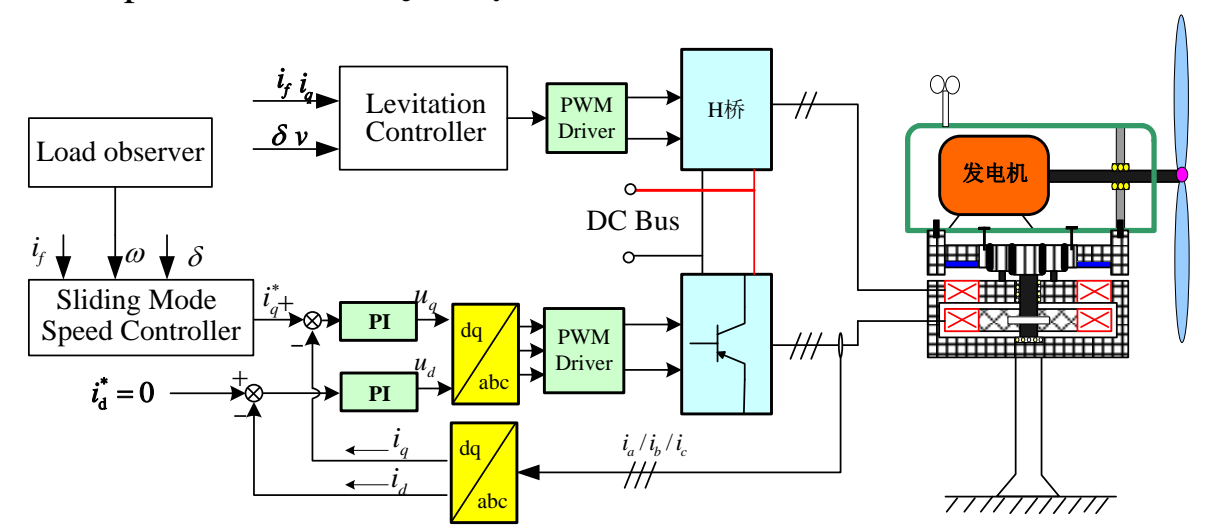

Figure 3. Magnetic levitation yaw system control strategy map

Define the candidate Lyapunov function as:

$$
V=\frac{1}{2} s^{2}
$$

Define the control input as:

$$
\begin{gathered}
u=\frac{2 J}{3 n_{p} L_{m} i_{f}}\left[\frac{T_{L}}{J}+\dot{\omega}_{\text {ref }}-c \omega+c \omega_{\text {ref }}-\frac{1}{c} \eta \operatorname{sgn}(s)\right] \\
\text { Due to } \quad V=\frac{1}{2} s^{2} \geq 0 \quad(\text { when } s=0, \dot{V}=0) \quad \dot{V}=s \dot{s}=-\eta|s| \leq 0
\end{gathered}
$$

So the control system must be stability, which can adjust the $c 、 \eta$ to change the tracking performance.

\subsection{Load Observer Design}

By analyzing the control input (17), $T_{L}$ is unknown which will affect the control effect, for a continuous sampling system, the load variation is not abrupt at each moment, and the load can be seen as a constant variable. Assuming that the load $T_{L}$ is a constant at control period, so $\dot{T}_{L}=0$, the equation can be obtained as follows: 


$$
\left[\begin{array}{c}
\dot{\omega}_{r} \\
\dot{T}_{L}
\end{array}\right]=\left[\begin{array}{cc}
0 & -\frac{1}{J} \\
0 & 0
\end{array}\right]\left[\begin{array}{l}
\omega_{r} \\
T_{L}
\end{array}\right]+\left[\begin{array}{c}
\frac{1}{J} \frac{3}{2} n_{p} L_{m} i_{f} \\
0
\end{array}\right] i_{q}
$$

Define the load observer:

$$
\dot{\hat{T}}_{L}=\dot{T}_{L}+L_{1}\left(\dot{\omega}_{r}-\dot{\hat{\omega}}_{r}\right)
$$

Take the torque equation (19) into the equation (20), the load observer [8] [9] can be described as

$$
\dot{\hat{T}}_{L}=L_{1}\left(\dot{\omega}_{r}+\frac{1}{J} \hat{T}_{L}-\frac{1}{J} \frac{3}{2} n_{p} L_{m} i_{f} i_{q}\right)
$$

In view of the fact that there lie much more high-frequency interferences in the actual condition, the new variables can be further expressed as:

$$
\dot{x}_{c 1}=L_{1}\left(\frac{1}{J} T_{L}-\frac{1}{J} \frac{3}{2} n_{p} L_{m} i_{f} i_{q}\right)
$$

Remarks: The load observer for the actual load can be achieved by the reasonable selection of the load observer gain $L_{1}$, so the load observer is designed $\dot{\hat{T}}_{L}=L_{1} \dot{\omega}_{r}+\dot{x}_{c 1}$.

\section{Simulation Experiment}

Based on MATLAB/ Simulink simulation experiment platform, a magnetic suspension yaw system based on the disc motor is built. The constant speed control of the disc motor under one constant air gap is realized. The results of the simulation experiment are as follows:
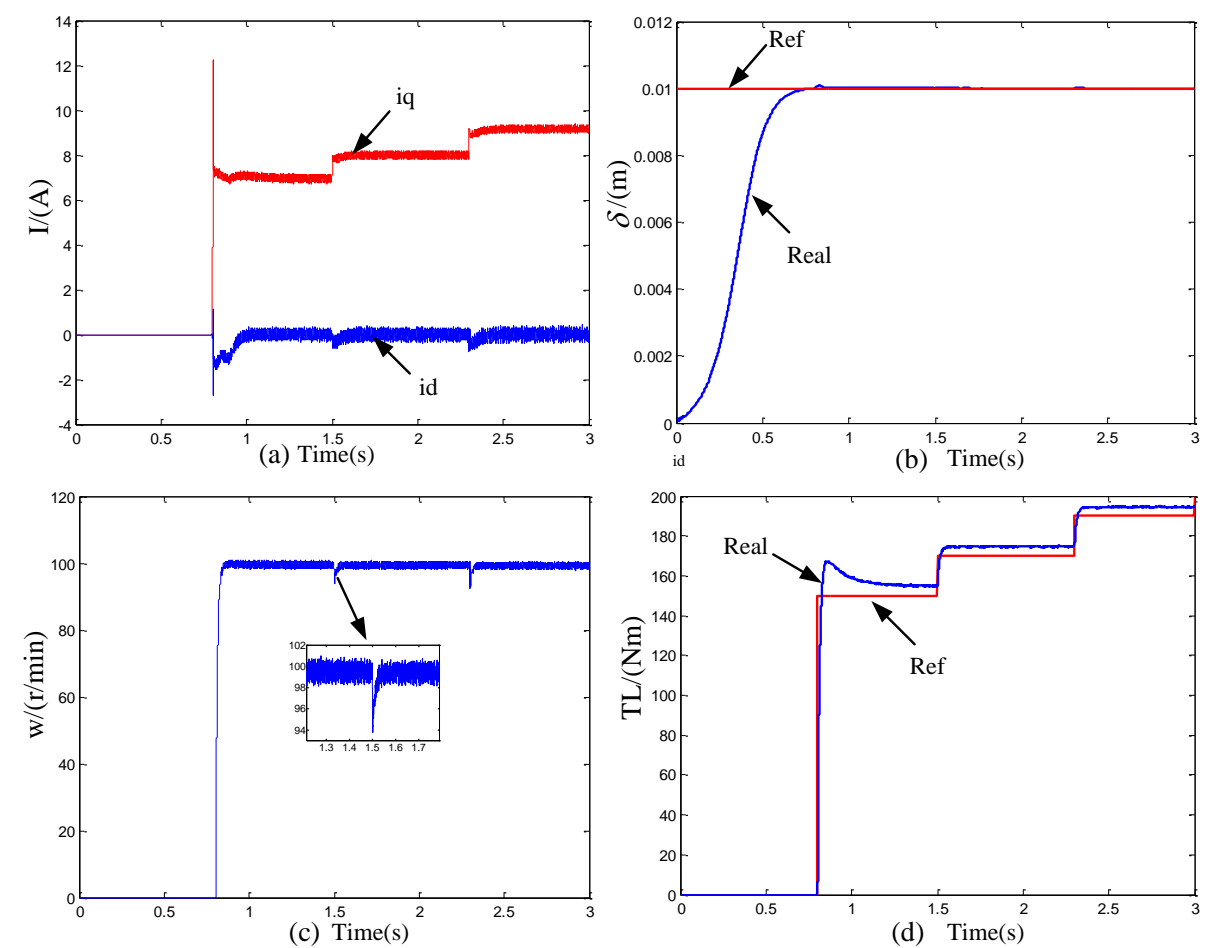

Figure 4. Simulation results of magnetic levitation yaw system

Figure (a) for the current of the disc motor iq and id, observed in the figure found that it only took $0.2 \mathrm{~s}$ to maintain a steady-state operation, the maximum impact current iq was $12 \mathrm{~A}$, the current increased with the load increased gradually, the id steady-state value fluctuated up and down around 0 , and to ensure the speed tracking performance the current changed fast.

Figure (b) for the suspension air gap, at time of 0 the nacelle began to suspension, in the time of $0.6 \mathrm{~s}$ achieved a constant air gap reference $0.01 \mathrm{~m}$, in the time of $0.8 \mathrm{~s}$, due to the starting up of the yaw action, the air gap fluctuated, but it only fluctuated under the range of $10^{\wedge}-4 \mathrm{~m}$.

Figure (c) for the speed of the disc motor, in the time of $0.8 \mathrm{~s}$ the yaw start, the rise time was only $0.05 \mathrm{~s}$, and in the process of rising was strictly no overshoot, in the stable operation, speed fluctuation was only $2 \mathrm{r} / \mathrm{min}$, with 
the load increased the speed appeared a sudden drop, the descending speed only up to 8r/min, and with function of the control input the speed rise quickly, the rise time was only $0.15 \mathrm{~s}$.

Figure (d) shows the observed load and the actual load changed, found that the observed load changing lagged behind the actual load, but the lag time was only $0.1 \mathrm{~s}$, at the starting moment of the motor, because the load observer was based on the speed changed, so in the time of $0.9 \mathrm{~s}$ the observed load had a large overshoot, the design of the observer not only to achieve the function of load observe, but compensate the speed effectively.

By analyzing the simulation results, it can be seen that the magnetic suspension yaw system can achieve a constant speed control under the suspended state. The precise yawing of the magnetic levitation machine solves the problem of large yaw loss in the traditional yaw system and reduces the later maintenance cost greatly, which is of practical significance.

\section{References}

[1]. L.Fingersh, M.Hand, A. Laxson. Wind Turbine Design Cost and Scaling Model. Technical Report NREL/TP-500-40566 December 2006.

[2]. Cui W, Yu F, Liu X, et al. Analysis of the passive yaw mechanism of small horizontal-axis wind turbines[C] World Non-Grid-Connected Wind Power and Energy Conference, 2009. WNWEC 2009. IEEE, 2009:1-5.

[3]. Yoshifumi Nishizawa,1 Hideki Tokuyama,2 Yuichi Nakajo,1 Yaw Behavior of Horizontal-Axis Small Wind Turbines in an Urban Area, WIND ENGINEERING VOLUME 33, NO. 1, 2009, PP 19-30.

[4]. M. Maggiore and R. Becerril, Modeling and control design for a magnetic levitation system[J], International Journal of Control, 2004, 77(10):964-977.

[5]. X. Shan and C. H. Menq, Robust disturbance rejection for improved dynamic stiffness of a magnetic suspension stage [J], IEEE Transactions on Mechatronics, 2002, 7(3):289-295.

[6]. J. Yang, S. Li, and X. Yu, "Sliding-mode control for systems with mismatched uncertainties via a disturbance observer.” IEEE Trans. Ind. Electron. vol. 60, no. 1, pp. 3062-3068, Jan. 2013.

[7]. D. Ginoya, P. D. Shendge, and S. B, "Sliding mode control for mismatched uncertain systems using an extended disturbance observer," IEEE Trans. Ind. Electron., vol. 61, no. 4, pp. 1983-1992, Apr. 2014.

[8]. Gulati N, Barth E J. A Globally Stable, Load-Independent Pressure Observer for the Servo Control of Pneumatic Actuators [J]. IEEE/ASME Transactions on Mechatronics, 2009, 14(3):295-306.

[9]. Lin C K, Liu T H, Yang S H. Nonlinear position controller design with input-output linearization technique for an interior permanent magnet synchronous motor control system [J]. 2008, 1(1):14-26. 